\title{
Evaluating the efficacy of best practice alerts to improve Clostridium difficile early detection in hospital settings: A 6-month interim analysis of the 2-year prospective study
}

\author{
Ahmad Kaako
}

Department of Medicine, Mercy Hospital, Fort Smith, AR, USA

\begin{tabular}{|c|}
\hline Access this article online \\
\hline Website: www.avicennajmed.com \\
\hline DOI: 10.4103/ajm.AJM_85_17 \\
\hline Quick Response Code: \\
\hline$\square$
\end{tabular}

\begin{abstract}
Introduction: Clostridium difficile infection (CDI) is a leading cause of hospital-associated gastrointestinal illness with high burden on the health-care system. Design: Observational, retrospective, prospective, pre- and post-intervention study on the incidence and prevalence rates of CDIs. The primary outcome is the healthcare-onset CDIs (HO-CDIs) incidence rate and proportion. Methods: Best practice alerts (BPAs) for CDI were developed in electronic medical records and released on July 13,2016 . When high-risk patients for CDI are identified, BPAs are triggered requiring nurses to order contact isolation and stool studies followed by other subsequent BPAs encouraging providers to de-escalate antibiotics and order probiotics. Data on admission count, patient-days, CDIs categories prevalence, and incidence rates were collected for the 24-month preintervention and will be collected for the 24-month postintervention period. Results: An interim analysis comparing the data from the first 6-month postintervention period with the average 6-month data of the preintervention period showed that short-term BPAs use is associated with significant increase in the overall $C D I$ infection rate $(71.47 \mathrm{vs}$. $38.38, P<0.0001)$, incidence rate (53.81 vs. $28.76, P<0.0001)$, overall prevalence rate $(1.86$ vs. $1.32, P=0.001)$, and admission prevalence rate $(1.10$ vs. $0.70, P<0.0001)$. Despite the observed overall shift from $\mathrm{HO}$ toward $\mathrm{CO}$; no statistically significant difference in the HO-CDI event number and proportion was detected (28 [21.4\%] vs. 22 [29.1\%], $P=0.094)$. Conclusion: Short-term BPAs use is associated with significant increase in the overall CDI incidence and prevalence along with a non-statistically significant decline in HO-CDI proportion. Final analysis with full sample size is essential to provide a better picture about the long-term effect.
\end{abstract}

Key words: Best practice alert, Clostridium difficile, hospital-acquired infection

\section{INTRODUCTION}

Clostridium difficile infection (CDI) is a leading cause of hospital-associated gastrointestinal illness with associated significant impact on patients' morbidity and mortality. In the United States (US), CDI was estimated to be responsible for almost half a million infections and associated with approximately 29,000 deaths in $2011 .^{[1]}$ It places a high burden on the US health-care system and hospitals length of stay. Centers for Medicare and Medicaid Services (CMS)

Address for correspondence: Dr. Ahmad Kaako,

Department of Medicine, Mercy Hospital, Physicians Building,

7300 Rogers Ave, Fort Smith, AR, USA.

E-mail: ahmadkaako@hotmail.com requires all positive CDI tests be reported to the National Healthcare Safety Network (NHSN). CDI is adopted by CMS as a new outcome measure in the hospital value-based purchasing program, which adjusts CMS hospitals' payments effective January 2017. ${ }^{[2]}$

This is an open access journal, and articles are distributed under the terms of the Creative Commons Attribution-NonCommercial-ShareAlike 4.0 License, which allows others to remix, tweak, and build upon the work non-commercially, as long as appropriate credit is given and the new creations are licensed under the identical terms.

\section{For reprints contact: reprints@ @medknow.com}

Cite this article as: Kaako A. Evaluating the efficacy of best practice alerts to improve Clostridium difficile early detection in hospital settings: A 6-month interim analysis of the 2-year prospective study. Avicenna J Med 2018;8:87-91. 
NHSN uses LabID Event reporting system, which is a much less labor-intensive standardized method, based almost exclusively on laboratory data and limited admission date data, including patient care location without clinical evaluation of the patient. It provides proxy infection measures of $C$. difficile health-care acquisition, exposure burden, and infection burden. Although this system is not highly specific to capture true active infection versus colonization and does not identify the location where the infection was truly acquired, it is still a practical simplified useful proxy for CDIs.

For NHSN reporting purposes, CDI diagnosis is established with any CDI-positive laboratory assay (reported as an event) irrespective of the signs, symptoms, and clinical presentation. ${ }^{[3]}$ Categorization is based strictly on the number of hospital days between the specimen collection date and the date the patient is admitted to the facility irrespective of the clinical information

- $\leq 3$ days = community onset $(\mathrm{CO})$

- $\quad \geq 4$ days $=$ hospital onset $(\mathrm{HO})$.

The crucial clinical and financial impact of CDI on patients, hospitals, and health system resources has driven clinician, hospitals, and public health officials to collaborate to reduce the burden of CDIs and to monitor and correctly identify the onset of CDIs.

\section{Intervention}

Our health system uses EPIC electronic medical records (EMR) across all its facilities. It is an integrated health-care software suite which combines chart review, order management, documentation for inpatient and outpatient care, and many other systems for laboratory professionals, pharmacists, radiologists, and physicians. Using EMR data, high-risk patients for CDI are identified on admission if they have two or more of the following risk factors:

1. Previous history of CDI

2. Admission to a health-care facility in the previous 90 days

3. Antibiotic use during the previous 90 days.

Although there are many risk factors for $C$. difficile in the literature, the above risk factors were prioritized for inclusion by the infectious disease and gastroenterology expert opinion serving on the designated committee. Automated clinical decision support tool was developed in EMR to display best practice alerts (BPAs) for nursing staff and providers aiming to identify high-risk patients in the targeted timeframe. Both BPA protocols were successfully piloted in a central facility and then expanded to all Mercy facilities nationwide. Verbal teaching sessions and online education assignments were done for the nursing staff to raise awareness about CDI impact and be familiar with the protocol. All the BPAs went live on Monday, July 13, 2016, in the entire Mercy Health System.

Nursing CDI protocol was developed to be applied during hospital days 1-3. When a high-risk patient is identified on admission, the nurse will receive BPA-1 querying if the patient has had 3 or more unformed stools in the previous $24 \mathrm{~h}$. If the condition is met, the nurse is prompted to collect a stool for CDI testing and start enteric contact isolation ordered directly from the BPA alert. If not met, EMR settings will continue to monitor stool documentations in the high-risk patients, and BPA- 2 will be triggered when three or more unformed stools have been documented within the first three days of admission.

Providers CDI Protocol was developed to be displayed on the fourth day of admission. When a high-risk patient has documented diarrhea without a laxative or bowel preparation order, the provider will receive BPA-3 prompting to order CDI testing and enteric contact isolation, otherwise, the reason for acknowledgment is required to dismiss the alert. If a high-risk patient with no diarrhea is placed on antibiotics, the provider will receive BPA- 4 alert on the third day of the antibiotic course encouraging to de-escalate antibiotics, order probiotics, and consider suppressive oral metronidazole or oral vancomycin.

\section{METHODS}

After obtaining approval from our Central Institutional Review Board, we have conducted retrospective, and prospective cohort study in Mercy Hospital Fort Smith aimed to evaluate the burden of CDIs in our facility and to determine whether BPAs can help in the early detection of inpatient $\mathrm{CDI}$ and the reduction of inpatient $\mathrm{HO}$-CDIs rates.

Pre- and post-BPA implementation periods were selected to be two years before and after BPA release date on July 13, 2016, respectively, as shown in Figure 1.

\section{Data collection}

We have applied the LabID Event reporting methodology, definitions, and categorization [Table 1]. Pre-BPA period data were collected including number of admissions, patient days, and the overall facility-wide positive CDI events. Incident inpatients CDI cases were identified. Duplicate results were removed. Incidence rates for CDI categories per 10,000 inpatient days and prevalence rates per $100 \mathrm{admissions}$ were calculated per CDC/NHSN Surveillance Definitions. ${ }^{[3]}$ 


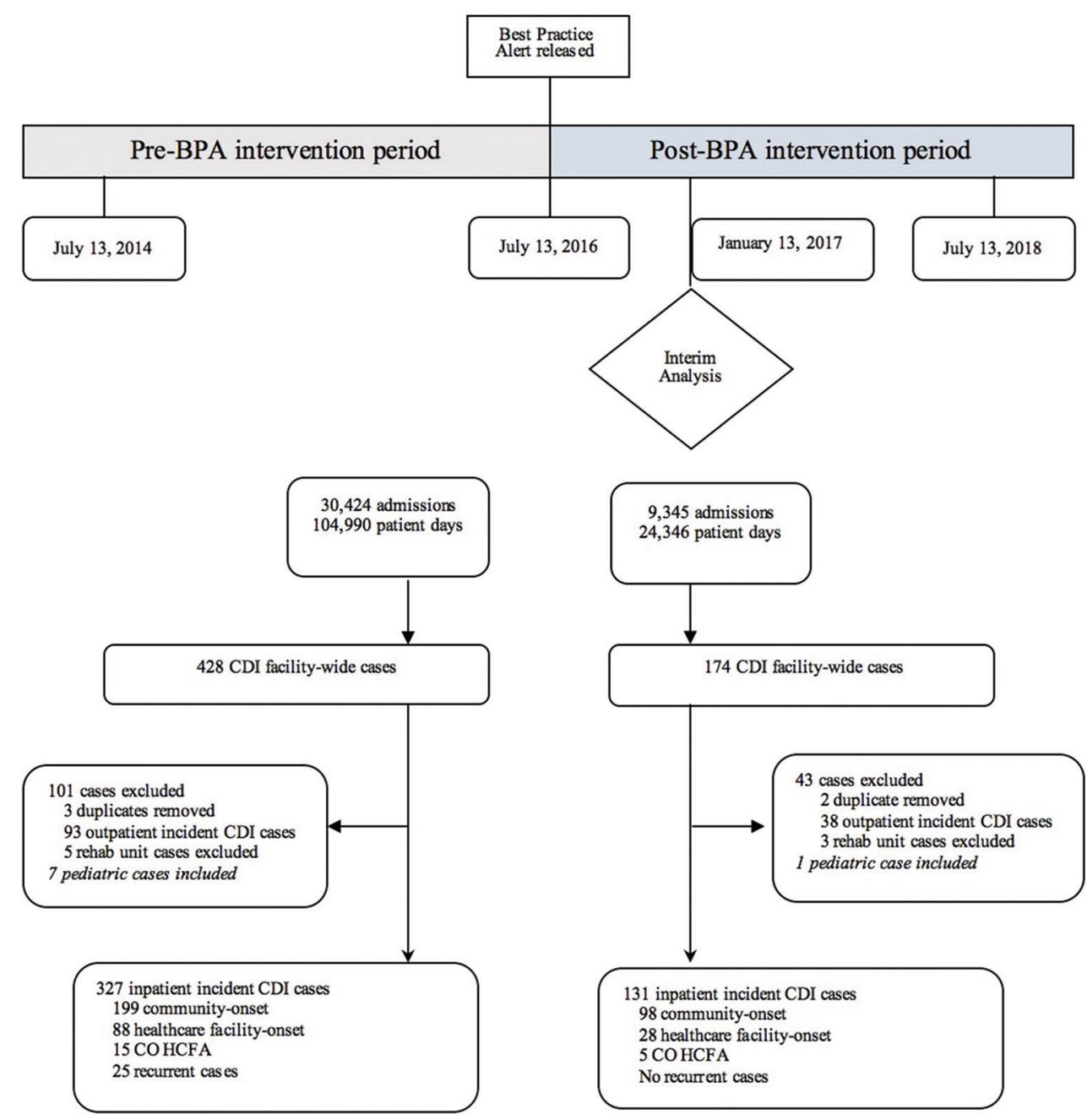

Figure 1: Schematic timeline and data collection flow for the best practice alerts intervention study. CDI: Clostridium difficile infection, CO-HCFA: Community-onset healthcare facility-associated

As NHSN reporting system includes pediatric cases, we have included them in our analysis to be consistent with the standardized method of reporting. The same data collection process was utilized for the first 6-month post-BPA period.

\section{Statistical analysis}

In our interim analysis, two-sample tests of proportions were conducted to compare pre- and post-BPA data using contingency tables. Pearson $\chi^{2}$-test was used to compare proportions for appropriate cell count tables. Fisher's exact test was used for small table cell size $(n<5) . P<0.05$ is considered statistically significant. All calculations were made using STATA version 14 (StataCorp, College Station, TX, USA).

\section{RESULTS}

For 24 months before BPA implementation, 327 inpatient incident CDI cases out of total 428 facility-wide cases were detected (199 CO [60.3\%], $88 \mathrm{HO}$ [27.2\%], 25 [7.8\%] recurrent and 15 CO-HCFA [5\%]) among 30,424 admission and 104,990 patient days. Interim analysis for the first 6-month period after the BPA implementation, 130 inpatient incident CDI cases out of total 174 facility-wide cases were detected (98 CO [74.8\%], $28 \mathrm{HO}$ [21.4\%], and 5 CO-HCFA [3.8\%]) among 9345 admissions and 24,346 patient days [Table 2].

\section{DISCUSSION}

In our interim analysis, we have observed higher CDIs incidence and prevalence rates in the early post-BPAs phase. One explanation for this rise could be improved detection using BPA.

For further evaluation, an A3 problem-solving process is being developed by our multidisciplinary teams to give more insight after root cause analysis. Consideration for areas of improvement includes compliance with hand hygiene, isolation precautions, antibiotics stewardship, 


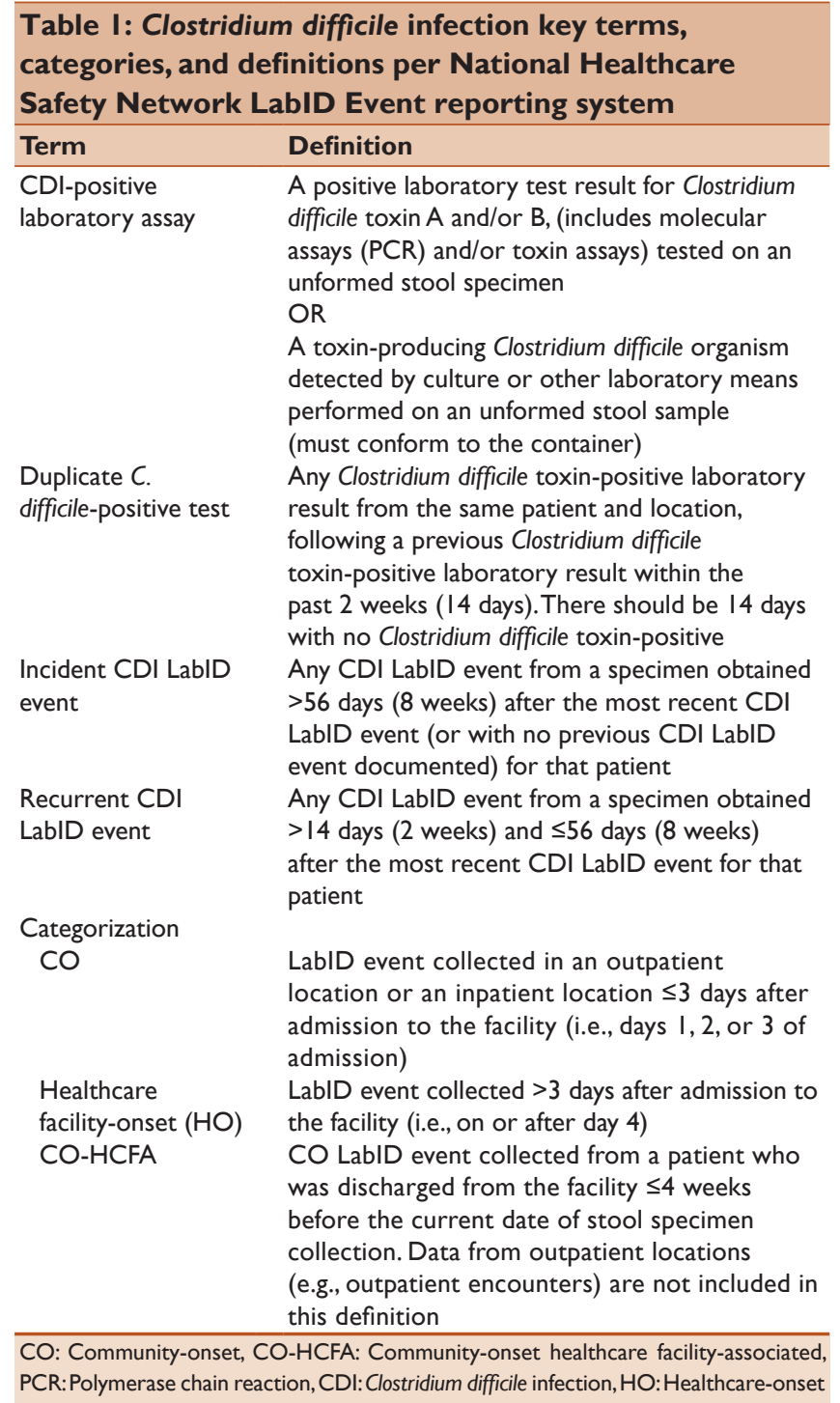

isolating high-risk patients on admission, and raising environmental services staff awareness. The observed high CO-CDI proportion (almost 75\%) is consistent with the recent shift in CDI epidemiology over the last decade showing that CO-CDI rates are dramatically increasing. ${ }^{[4]}$ Raising public and patients awareness is a crucial step in CDIs control and prevention.

Our interim analysis has limitations. Failure to detect a significant change in the HO-CDI proportion between the two groups may have been due to our small interim analysis postintervention sample size. We were not able to measure the exact role of BPAs on the observed higher incidence and prevalence as it could be related to nonmeasured confounders in our study, such as antibiotics prescribing behavior, staff awareness level about CDIs and their compliance with enteric isolation precautions before and after the intervention. Nevertheless, our observation of a significant increase in the overall incidence and prevalence rate may need further studies designed to measure all the potential confounders, which is not our study preliminary outcome.

We have not included the patients' demographic and clinical characteristics in this brief report as we are planning to present the big picture at the end of our study.

This study highlights the importance of EMR and BPAs utilization as useful tools to enhance our understanding and management of diseases. Previous studies have utilized EMR and BPAs to risk stratify $\mathrm{HO}-\mathrm{CDIs},{ }^{[5]}$ predict the risk for (HO-CDI) based on risk score ${ }^{[6]}$ and to help with providing more standardized management. ${ }^{[7]}$

\begin{tabular}{|c|c|c|c|}
\hline & Average 6 months' preintervention & 6 months' Postintervention & $\mathbf{P}$ \\
\hline \multicolumn{4}{|l|}{ Denominator data } \\
\hline Patient days $(n)$ & 26248 & 24346 & $<0.001$ \\
\hline Admission (n) & 7606 & 9345 & \\
\hline \multicolumn{4}{|l|}{ Numerator data } \\
\hline All positive CDI (n) & 107 & 174 & 0.011 \\
\hline Outpatient, $n(\%)$ & $23.25(21.7)$ & $38(22.2)$ & \\
\hline Inpatient incident, $n$ (\%) & $75.5(70.6)$ & $13 \mid(75)$ & \\
\hline Inpatient recurrent, $n$ (\%) & $6.25(7.8)$ & 0 & \\
\hline Other, $n(\%)$ & $2(1.9)$ & $5(2.8)$ & \\
\hline Inpatient incident cases & 75.5 & $|3|$ & 0.080 \\
\hline $\mathrm{CO}, \mathrm{n}(\%)$ & $49.75(60.3)$ & $98(74.8)$ & 0.066 \\
\hline $\mathrm{HO}, n(\%)$ & $22(27.2)$ & $28(21.4)$ & 0.094 \\
\hline CO-HCFA, $n(\%)$ & $3.75(5)$ & $5(3.8)$ & 0.600 \\
\hline Overall prevalence rate & 1.32 & 1.86 & 0.001 \\
\hline Admission prevalence rate & 0.70 & 1.10 & $<0.001$ \\
\hline CDI rate & 38.38 & 71.47 & \\
\hline CDI incidence rate & 28.76 & 53.81 & $<0.001$ \\
\hline $\mathrm{HO}$ incidence rate & 8.38 & 11.50 & 0.143 \\
\hline $\begin{array}{l}\text { Facility CDI combined } \\
\text { incidence rate }\end{array}$ & 9.81 & 13.55 & 0.104 \\
\hline
\end{tabular}


In summary, we conclude that short-term BPAs use is associated with significant increase in the overall CDI infection rate, incidence rate, overall prevalence rate, and admission prevalence rate along with a non-statistically significant decline in HO-CDI proportion. Final analysis with full sample size is essential to provide a better picture about the long-term effect.

\section{Financial support and sponsorship}

Nil.

\section{Conflicts of interest}

There are no conflicts of interest.

\section{REFERENCES}

1. Lessa FC, Mu Y, Bamberg WM, Beldavs ZG, Dumyati GK, Dunn JR, et al. Burden of Clostridium difficile infection in the United States. N Engl J Med 2015;372:825-34.
2. Centers for Medicare and Medicaid Services. Fiscal Year (FY) 2016 Results for the CMS Hospital Value-Based Purchasing Program; 2015. Available from: https://www.cms.gov/Newsroom/MediaReleaseDatabase/Fact-sh eets/2015-Fact-sheets-items/2015-10-26.html. [Last accessed on 2018 Apr 19].

3. Centers for Disease Control and Prevention. CDC/NHSN Surveillance Definitions for Specific Types of Infections; 2016. Available from: http:// www.cdc.gov/nhsn/pdfs/pscmanual/17pscnosinfdef_current.pdf. [Last accessed on 2018 Apr 19].

4. Gupta A, Khanna S. Community-acquired Clostridium difficile infection: An increasing public health threat. Infect Drug Resist 2014;7:63-72.

5. Zilberberg MD, Tabak YP, Sievert DM, Derby KG, Johannes RS, Sun X, et al. Using electronic health information to risk-stratify rates of Clostridium difficile infection in US hospitals. Infect Control Hosp Epidemiol 2011;32:649-55.

6. Tabak YP, Johannes RS, Sun X, Nunez CM, McDonald LC. Predicting the risk for hospital-onset Clostridium difficile infection (HO-CDI) at the time of inpatient admission: HO-CDI risk score. Infect Control Hosp Epidemiol 2015;36:695-701.

7. Revolinski S. Implementation of a clinical decision support alert for the management of Clostridium difficile infection. Antibiotics (Basel) 2015;4:667-74. 